\title{
A Kernel $k$-Means-based Method for Diabetes Diagnosis
}

\author{
Tru $\mathrm{CAO}^{1,2}$, Chau VO ${ }^{1}$, Son NGUYEN ${ }^{1}$, Atsushi INOUE ${ }^{2}$, Duanning ZHOU ${ }^{2}$ \\ ${ }^{1}$ Ho Chi Minh City University of Technology, Vietnam National University, Ho Chi Minh City, Vietnam \\ tru@cse.hcmut.edu.vn,chauvtn@hcmut.edu.vn,tsonnguyen95@gmail.com \\ ${ }^{2}$ Eastern Washington University, Spokane, Washington State, United States of America \\ tcao2@ewu.edu,ainoue@ewu.edu,dzhou@ewu.edu
}

\begin{abstract}
Diabetes diagnosis is important due to the death and complication consequences caused by the disease. It thus has attracted much research attention and effort in Artificial Intelligence to support human decisions. Our work proposes a kernel $k$-means-based predictive method and explores attribute selections for effective and robust diabetes diagnosis. This method uses homogeneous subclusters in the high dimensional kernelized feature space to compute the distance of a new instance to those subclusters and classify it accordingly. The PIMA and MIMIC data sets are respectively used for training and testing. Our experimental results could identify the best effective attribute groups and show that the proposed method outperforms existing ones for the task.
\end{abstract}

Keywords: Disease diagnosis, feature selection, clustering, PIMA data set, MIMIC database

\section{INTRODUCTION}

As reported in [20], diabetes is considered to be the seventh leading cause of death in 2030. It has risen from 108 million diabetic people in 1980 to 422 million ones in 2014. It is also a major cause of blindness, kidney failure, heart attacks, stroke, and lower limb amputation.

Diabetes diagnosis is thus important and has attracted much research effort in Artificial Intelligence to support doctors' decisions, as recently surveyed in [4]. In particular, machine learning methods were proposed, such as decision trees [6, 19], $k$-nearest neighbors [1], logistic regression [10, 14], neural networks [3, 5, 6, 7, 10, 11, 17], and support vector machines $[12,15]$. In [2], electronic medical records were exploited for personalized diabetes management.

Besides prediction, $[6,8,16]$ provided interpretable rules for decision making support in the disease diagnosis. Also, most of the above-mentioned works combined machine learning with other methods for data preprocessing such as genetic method [3], self-organizing maps [11], $k$-means [12], fuzzy c-means [16], random forests [14], and principal component analysis [11, 19].

Consequently, there were many different groups of attributes (i.e., feature) used for diabetes diagnosis as a machine learning task. However, to the best of our knowledge, there has been no extensive examination of attribute groups to identify the most effective ones for prediction. Meanwhile, doctors have relied on their medical knowledge to examine a patient's clinical and paraclinical information to diagnose diabetes.

In this paper, we propose a novel method and explore various attribute groups for diabetes diagnosis. Our method is a kernel $k$-means-based one that classifies a new instance, i.e., as positive or negative, depending on its distances to the homogeneous subclusters obtained from a training dataset in a high dimensional kernelized feature space. We use the PIMA dataset [17] for training our model and diabetes cases from the MIMIC database [9] for testing it in comparison to some existing ones.

Next, Section 2 formulates the diabetes diagnosis problem and presents our proposed method. Section 3 presents our experiments evaluating the effectiveness of different attribute groups and the performance of the proposed method. Finally, Section 4 gives some concluding remarks.

\section{THE PROPOSED METHOD}

\subsection{Problem definition}

Given the information of a person, the problem is to predict if the person potentially has diabetes. The information includes his/her lab test results, current and past personal diseases, medication history, and family medical history. The prediction is binary, i.e., 1 if positive with diabetes, or 0 if negative.

\subsection{Attribute groups}

We summarize and name the attribute groups used in the corresponding works based on the attributes in the 
PIMA data set, as presented in Table 1 below. The description of each attribute can be found in [17].

Group 8 was originally proposed for diabetes diagnosis in [17]. Group 2 in [6] was found in the extracted rules. Group 3_1 in [14] contained the top ranked attributes. Group 6_2 in [1] was the best case among several selected groups. Especially, Group 5 is what doctors rely on to diagnose diabetes, as provided in the decision support medical information visualization system [13].

Table 1: Different attribute groups in the existing works

\begin{tabular}{|c|c|c|l|}
\hline $\begin{array}{c}\text { Related } \\
\text { Work }\end{array}$ & $\begin{array}{c}\text { Named } \\
\text { Group }\end{array}$ & $\begin{array}{c}\text { Number of } \\
\text { Attributes }\end{array}$ & \multicolumn{1}{|c|}{ Attributes } \\
\hline$[6]$ & 2 & 2 & Glucose, BMI \\
\hline$[14]$ & $3 \_1$ & 3 & Glucose, Insulin, BMI \\
\hline$[15]$ & $3 \_2$ & 3 & Pregnancies, Glucose, Age \\
\hline$[3]$ & 4 & 4 & Glucose, Insulin, BMI, Age \\
\hline$[13]$ & 5 & 5 & $\begin{array}{l}\text { Glucose, BloodPressure, Insulin, BMI, } \\
\text { Age }\end{array}$ \\
\hline$[12]$ & $6 \_1$ & 6 & $\begin{array}{l}\text { Pregnancies, Glucose, Insulin, BMI, } \\
\text { DiabetesPedigreeFunction, Age }\end{array}$ \\
\hline$[1]$ & $6 \_2$ & 6 & $\begin{array}{l}\text { Pregnancies, Glucose, BloodPressure, } \\
\text { Insulin, BMI, DiabetesPedigreeFunction }\end{array}$ \\
\hline$[17]$ & 8 & 8 & $\begin{array}{l}\text { Pregnancies, Glucose, BloodPressure, } \\
\text { SkinThickness, Insulin, BMI, } \\
\text { DiabetesPedigreeFunction, Age }\end{array}$ \\
\hline
\end{tabular}

\subsection{The proposed kernel $\boldsymbol{k}$-means-based method}

Firstly, diabetes shares some symptoms and has common medical attribute values with some other diseases. This leads to data overlapping for diabetic people and non-diabetic people, as shown in [11] via visualization. Therefore, we propose to use a kernel to map the original diabetes data space to a higher dimensional one, so that instances can be more discriminated.

Secondly, in medical research, the $k$-nearest neighbor method has recently been used for personalized diabetes management in [2]. However, if $k$ is small, this method is ineffective with noises and, if $k$ is large, it causes class heterogeneity of the $k$ returned nearest neighbors. To overcome it, in this work we propose to use the 1-nearest neighbor method at the prediction step but on the derived homogeneous subclusters, rather than on the main clusters obtained from the training step using kernel $k$-means [18].

Details of our proposed method for diabetes prediction are as follows:

\section{Input:}

- $D_{t}$ is a training set of instances $X_{i}$ with classes $y_{i}$,

- $D_{u}$ is a set of new instances $X_{j}$,

- $k$ is a predefined number of clusters,

- sigma is a bandwidth of the Gaussian kernel function.

\section{Output:}

- Predicted class $y_{j}$ of $X_{j}$ for every $X_{j} \in D_{u}$.

\section{Process:}

Step 1: Construct a clustering model in the feature space of the training data

1.1. Compute a kernel matrix $K M$ from the instances in $D_{t}$ :

$$
\begin{aligned}
& K M\left(X_{r}, X_{q}\right)=K_{r q}=e^{-\frac{\left\|X_{r}-X_{q}\right\|^{2}}{2 * \operatorname{sigma} a^{2}}} \\
& \text { where }\left\|X_{r}-X_{q}\right\|=\sqrt{\sum_{d=1 . . p}\left(x_{r d}-x_{q d}\right)^{2}} \\
& \text { for } r=1 . . n_{t} \text { and } q=1 . . n_{t}
\end{aligned}
$$

1.2. Establish a membership matrix $\gamma$ between $k$ clusters $C_{o}$ and $n_{t}$ instances $X_{i}$ in $D_{t}$, for $o=1 . . k, i=1 . . n_{t}$. Each cell in $\gamma$ is 1 if $X_{i}$ belongs to $C_{o}$; or 0 , otherwise. This matrix is initialized with the traditional $k$-means method on $D_{t}$.

1.3. Repeat the following substeps until the membership matrix $\gamma$ is unchanged:

1.3.1. Update the distance between each cluster $C_{o}$ and each instance $X_{r}$ in $D_{t}$ in the feature space as follows:

$$
\left\|\phi\left(X_{r}\right)-C_{o}\right\|^{2}=K_{r r}-\frac{2 \sum_{q=1 . . n_{t}} \gamma_{o q} K_{r q}}{\sum_{q=1 . . n_{t}} \gamma_{o q}}+\frac{\sum_{v=1 . . n_{t}} \sum_{z=1 . . n_{t}} \gamma_{o v} \gamma_{o z} K_{v z}}{\sum_{v=1 . . n_{t}} \sum_{z=1 . . n_{t}} \gamma_{o v} \gamma_{o z}}
$$

for $o=1 . . k$ and $r=1 . . n_{t}$

1.3.2. Update the membership $\gamma_{\text {or }}$ between the instance $X_{r}$ and the cluster $C_{o}$ as follows:

$$
\begin{aligned}
& \gamma_{o r}=\left\{\begin{array}{c}
1, \text { if }\left\|\phi\left(X_{r}\right)-C_{o}\right\|^{2}=\operatorname{argmin}_{o^{\prime}=1 . . k}\left(\left\|\phi\left(X_{r}\right)-C_{o^{\prime}}\right\|^{2}\right) \\
0, \text { otherwise }
\end{array}\right. \\
& \text { for } r=1 . . n_{t} \text { and } o=1 . . k
\end{aligned}
$$

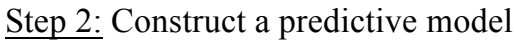

2.1. Derive homogeneous subclusters $H C_{o}$, from the main clusters $C_{o}$ in Step 1 for $o^{\prime}=1 . . k^{*}|Y|, o=1 . . k$

A homogeneous subcluster $H C_{o}$, is defined as a smaller group of the instances in $C_{o}$ that belong to the same class in $Y$. For each main cluster $C_{o}$, the maximum number of homogeneous subclusters $H C_{o}$, is $|Y|$ when only one instance in $C_{o}$ belongs to each class in $Y$. The minimum number is 1 when all of the instances in $C_{o}$ belong to the same class.

2.2. Assign the common class $y_{o}$, of all of the instances in a homogeneous subcluster $H C_{o}$, to that homogeneous subcluster $H C_{o}$, for $o^{\prime}=1 . . k^{*}|Y|$

Step 3: Predict a class $y_{j}$ of a new instance $X_{j}$ for every $X_{j}$ $\in D_{u}$

3.1. Calculate the kernel-induced Euclidean distance between $X_{j}$ and each homogeneous subcluster $H C_{o}$, via its mean vector: 
$\operatorname{distance}\left(X_{j}, H C_{o}{ }^{\prime}\right)=2-2 * K\left(X_{j}, H C_{o}\right)$

where $K()$ is the Gaussian kernel function, $j=1 . . n_{u}$, $o{ }^{\prime}=1 . . k^{*}|Y|$.

3.2. Based on the smallest distance in the previous substep, assign the class $y_{o}$, of the nearest homogeneous subcluster $H C_{o}$, to the new instance $X_{j}$ for prediction, i.e., $y_{j}=y_{o}$.

\section{EVALUATION}

The evaluation of our proposed method is to answer the following questions:

Question 1: Given a data set and a method, which attribute group is more effective for diabetes diagnosis?

Question 2: Given a data set and an attribute group, which method is more effective for diabetes diagnosis?

Question 3: Given a data set, which method is more robust to missing values of attributes of instances?

The effectiveness is measured by the Accuracy, Recall, Precision, and F measures. In this context, Accuracy is the percentage of correctly predicted people to be either positive or negative with diabetes. Recall is the percentage of all true diabetic patients that are predicted. Meanwhile, Precision is the percentage of positively predicted people who are truly diabetic patients.

The robustness is addressed because, without preprocessing, there are many instances in the training and testing data sets that miss some attribute values. Table 2 presents the details of the two data sets PIMA and MIMIC that we use for training and testing, respectively.

We compare the proposed method with the following typical baseline methods: $k$-nearest neighbors $(k-\mathrm{NN})$ [1], logistic regression (LR) $[10,14]$, neural networks (NN) [3, $5,6,7,10,11,17]$, and support vector machines (SVM) $[12,15]$.

Table 2: Data descriptions for training and testing

\begin{tabular}{|c|c|c|c|c|c|}
\hline $\begin{array}{c}\text { Data } \\
\text { set }\end{array}$ & Role & $\begin{array}{c}\text { Number of } \\
\text { Instances }\end{array}$ & $\begin{array}{c}\text { Percentage } \\
\text { of Positives }\end{array}$ & $\begin{array}{c}\text { Percentage } \\
\text { of Negatives }\end{array}$ & $\begin{array}{c}\text { Percentage of } \\
\text { Missing Values }\end{array}$ \\
\hline PIMA & Training & 768 & $34.9 \%$ & $65.1 \%$ & $12.1 \%$ \\
\hline MIMIC & Testing & 180 & $38.9 \%$ & $61.1 \%$ & $15.4 \%$ \\
\hline
\end{tabular}

For parameter settings, we use $k$ in $\{1,3,5\}$ for $k$-NN; feedforward multiplayer perceptrons with the default network configuration, learning rate $=0.01$, and training time $=1,000$ epochs for $\mathrm{NN}$; the radial basis function as a kernel with gamma $=0.01$ and $C=50$ for SVM. As for the proposed kernel $k$-means-based method (KK), we use the Gaussian kernel function with the sigma computed from the attribute variances in $\{0.1,0.09,0.08,0.07,0.06,0.05$, $0.04,0.03,0.02,0.01,0.009,0.008,0.007,0.006,0.005$, $0.004,0.003,0.002,0.001,0.0009,0.0008,0.0007$, $0.0006,0.0005,0.0004,0.0003,0.0002,0.0001\}$ and $k$ in $\{2,4,6,8,10,12,14\}$. The grid search scheme was used.

For the results, Accuracy is presented in percent (\%) in the range $[0,100]$, while Recall, Precision, and F-measure are shown in the range $[0,1]$. For Question 1, Table 3 and Table 4 respectively show the attribute group that gives a certain method the highest Accuracy or the highest F-measure. For each method and each attribute group, the highest measures are obtained with the best tuned parameters. In both cases, our proposed method has the best performance, with attribute group $3 \_1$.

Table 3: The highest Accuracy for each method

\begin{tabular}{|c|c|c|c|c|c|}
\hline Method & Attribute Group & Accuracy & Recall & Precision & F-measure \\
\hline KK & $3 \_1$ & $\mathbf{9 2 . 7 8}$ & 0.81 & 1.00 & 0.90 \\
\hline$k$-NN & 2 & 67.78 & 0.40 & 0.64 & 0.49 \\
\hline LR & 2 & 69.44 & 0.46 & 0.65 & 0.54 \\
\hline NN & $6 \_1$ & 71.11 & 0.41 & 0.73 & 0.53 \\
\hline SVM & $6 \_1$ & 69.44 & 0.44 & 0.66 & 0.53 \\
\hline SVM & 8 & 69.44 & 0.46 & 0.65 & 0.54 \\
\hline
\end{tabular}

Table 4: The highest F-measure for each method

\begin{tabular}{|c|c|c|c|c|c|}
\hline Method & Attribute Group & Accuracy & Recall & Precision & F-measure \\
\hline KK & $3 \_1$ & 92.78 & 0.81 & 1.00 & $\mathbf{0 . 9 0}$ \\
\hline$k$-NN & $3 \_2$ & 60.00 & 0.56 & 0.49 & 0.52 \\
\hline LR & 5 & 63.89 & 0.56 & 0.53 & 0.55 \\
\hline NN & $3 \_1$ & 67.78 & 0.49 & 0.61 & 0.54 \\
\hline SVM & 5 & 62.22 & 0.59 & 0.51 & 0.55 \\
\hline
\end{tabular}

For Question 2, Tables 5-7 respectively present the highest Accuracy, Recall, and F-measure of each method for each attribute group. For each measure, the highest values are obtained with the best tuned parameters. It shows that our proposed method outperforms all of the compared ones on every measure and every attribute group.

Note that group 3_1 consists of only three attributes, namely, Glucose, Insulin, and BMI. However, for our proposed method, it gives the same Recall as, but better Accuracy and F-measure than any other group, including group 8 (with 8 attributes) that doctors have used for diabetes diagnosis. For the other methods, it also give better results than, or comparable to, the other attribute groups. That is, machines can support doctors to have simpler but more accurate diagnosis. 
Table 5: The highest Accuracy of each method for each attribute group

\begin{tabular}{|c|c|c|c|c|c|}
\hline Attribute Group & KK & $\boldsymbol{k}$-NN & LR & NN & SVM \\
\hline 2 & $\mathbf{7 7 . 7 8}$ & 67.78 & 69.44 & 68.33 & 68.33 \\
\hline 3_1 & $\mathbf{9 2 . 7 8}$ & 67.22 & 68.33 & 67.78 & 67.78 \\
\hline 3_2 & $\mathbf{6 3 . 8 9}$ & 60 & 63.33 & 60 & 62.22 \\
\hline 4 & $\mathbf{9 1 . 6 7}$ & 65.56 & 66.67 & 67.78 & 64.44 \\
\hline 5 & $\mathbf{8 8 . 3 3}$ & 66.67 & 63.89 & 66.67 & 62.22 \\
\hline 6_1 & $\mathbf{7 9 . 4 4}$ & 62.78 & 68.89 & 71.11 & 69.44 \\
\hline 6_2 & $\mathbf{7 5 . 5 6}$ & 60.56 & 67.22 & 68.89 & 68.89 \\
\hline 8 & $\mathbf{7 4 . 4 4}$ & 63.89 & 67.78 & 70.56 & 69.44 \\
\hline
\end{tabular}

Table 6: The highest Recall of each method for each attribute group

\begin{tabular}{|c|c|c|c|c|c|}
\hline Attribute Group & $\mathbf{K K}$ & $\boldsymbol{k}$-NN & $\mathbf{L R}$ & $\mathbf{N N}$ & $\mathbf{S V M}$ \\
\hline 2 & $\mathbf{1 . 0 0}$ & 0.40 & 0.46 & 0.47 & 0.49 \\
\hline 3_1 & $\mathbf{1 . 0 0}$ & 0.39 & 0.46 & 0.49 & 0.49 \\
\hline 3_2 & $\mathbf{1 . 0 0}$ & 0.59 & 0.54 & 0.57 & 0.51 \\
\hline 4 & $\mathbf{1 . 0 0}$ & 0.34 & 0.51 & 0.44 & 0.54 \\
\hline 5 & $\mathbf{1 . 0 0}$ & 0.34 & 0.56 & 0.47 & 0.59 \\
\hline 6_1 & $\mathbf{1 . 0 0}$ & 0.14 & 0.41 & 0.41 & 0.44 \\
\hline 6_2 & $\mathbf{1 . 0 0}$ & 0.13 & 0.39 & 0.43 & 0.43 \\
\hline 8 & $\mathbf{1 . 0 0}$ & 0.30 & 0.44 & 0.41 & 0.46 \\
\hline
\end{tabular}

Table 7: The highest F-measure of each method for each attribute group

\begin{tabular}{|c|c|c|c|c|c|}
\hline Attribute Group & $\mathbf{K K}$ & $\boldsymbol{k}$-NN & LR & NN & SVM \\
\hline 2 & $\mathbf{0 . 7 8}$ & 0.49 & 0.54 & 0.54 & 0.54 \\
\hline 3_1 & $\mathbf{0 . 9 0}$ & 0.48 & 0.53 & 0.54 & 0.54 \\
\hline 3_2 & $\mathbf{0 . 6 0}$ & 0.52 & 0.54 & 0.53 & 0.51 \\
\hline 4 & $\mathbf{0 . 8 8}$ & 0.44 & 0.55 & 0.52 & 0.54 \\
\hline 5 & $\mathbf{0 . 8 4}$ & 0.44 & 0.55 & 0.52 & 0.55 \\
\hline 6_1 & $\mathbf{0 . 7 6}$ & 0.22 & 0.51 & 0.53 & 0.53 \\
\hline 6_2 & $\mathbf{0 . 7 3}$ & 0.19 & 0.48 & 0.52 & 0.52 \\
\hline 8 & $\mathbf{0 . 6 8}$ & 0.39 & 0.52 & 0.52 & 0.54 \\
\hline
\end{tabular}

Table 8 and Table 9 focus on the proposed method. For each attribute group, Table 8 presents the corresponding Recall, Precision, and F-measure of the proposed method when it achieves the highest Accuracy. Meanwhile, Table 9 presents the corresponding Accuracy, Recall, and Precision of the proposed method when it achieves the highest F-measure. The results show that the proposed method has the highest values for the Accuracy at the same time as for the F-measure. Also, they are with groups 3_1, 4, and 5, which consist of only 3 to 5 attributes.

For Question 3, although there are respectively 12.1\% and $15.4 \%$ of missing attribute values in the PIMA and MIMIC data sets, our proposed method still achieves high Accuracy and F-measure as compared to the baseline ones. This proves its robustness to such noises in the data.
Table 8: The Recall, Precision, and F-measure corresponding to the highest Accuracy of the proposed method for each attribute group

\begin{tabular}{|c|c|c|c|c|}
\hline Attribute Group & Accuracy & Recall & Precision & F-measure \\
\hline 2 & 77.78 & 1.00 & 0.64 & 0.78 \\
\hline 3_1 & $\mathbf{9 2 . 7 8}$ & 0.81 & 1.00 & 0.90 \\
\hline 3_2 & 63.89 & 0.60 & 0.54 & 0.56 \\
\hline 4 & 91.67 & 0.79 & 1.00 & 0.88 \\
\hline 5 & 88.33 & 0.81 & 0.88 & 0.84 \\
\hline 6_1 & 79.44 & 0.80 & 0.71 & 0.75 \\
\hline 6_2 & 75.56 & 0.86 & 0.64 & 0.73 \\
\hline 8 & 74.44 & 0.60 & 0.70 & 0.65 \\
\hline
\end{tabular}

Table 9: The Accuracy, Recall, Precision corresponding to the highest F-measure of the proposed method for each attribute group

\begin{tabular}{|c|c|c|c|c|}
\hline Attribute Group & Accuracy & Recall & Precision & F-measure \\
\hline 2 & 77.78 & 1.00 & 0.64 & 0.78 \\
\hline $3 \_1$ & 92.78 & 0.81 & 1.00 & $\mathbf{0 . 9 0}$ \\
\hline $3 \_2$ & 58.89 & 0.79 & 0.48 & 0.60 \\
\hline 4 & 91.67 & 0.79 & 1.00 & 0.88 \\
\hline 5 & 88.33 & 0.81 & 0.88 & 0.84 \\
\hline 6_1 & 76.67 & 0.94 & 0.63 & 0.76 \\
\hline 6_2 & 75.56 & 0.86 & 0.64 & 0.73 \\
\hline 8 & 71.67 & 0.76 & 0.61 & 0.68 \\
\hline
\end{tabular}

\section{CONCLUSIONS}

In this paper, diabetes diagnosis has been studied as a binary classification task with our new proposed method. The method generates the clusters of similar instances in a high dimensional feature space using the Gaussian kernel function. Homogeneous subclusters are derived from the main clusters. A new instance is then predicted to belong to the class of the instances in its nearest subcluster.

Besides, our work has also conducted the experiments with several attribute groups used the related works and one used by medical doctors in practice. A new diabetes data set from the MIMIC database has been built as a testing data set for evaluating these attribute groups and the compared methods. The results have showed that the proposed method outperforms the others on every attribute group, and that the group of 3 attributes Glucose, Insulin, and BMI are sufficient for diabetes diagnosis.

For future work, a parameter-free version of the proposed method is considered for its stability and friendliness in practical use. Enhancing a medical information visualization system with the findings of this work is also of our interest. Such an enhancement is helpful for bridging a gap between human experts and computer systems in disease diagnosis. 


\section{REFFERENCES}

[1] S. Bahramian and A. Nikravanshalmani; Hybrid Method Based on K-Nearest-Neighbor Method and Adaboost with Selection of Feature by Genetic Methods for the Diagnosis of Diabetes, International Journal of Mechatronics, Electrical and Computer Technology, 6 (21), pp. 2977-2986, 2016.

[2] Dimitris Bertsimas, Nathan Kallus, Alexander M. Weinstein and Ying Daisy Zhuo; Personalized Diabetes Management Using Electronic Medical Records, Diabetes Care, 40, pp. 210-217, 2017.

[3] Dilip Kumar Choubey and Sanchita Paul; GA_RBF NN: a Classification System for Diabetes, International Journal of Biomedical Engineering and Technology, 23 (1), pp. 71-93, 2017.

[4] Dilip Kumar Choubey and Sanchita Paul; Classification Techniques for Diagnosis of Diabetes: a Review, International Journal Biomedical Engineering and Technology, 21 (1), pp. 15-39, 2016.

[5] Okan Erkaymaz, Mahmut Ozer and Matjaž Perc; Performance of Small-world Feedforward Neural Networks for the Diagnosis of Diabetes, Applied Mathematics and Computation, 311, pp. 22-28, 2017.

[6] Yoichi Hayshi and Shonosuke Yukita; Rule Extraction Using Recursive-Rule Extraction Method with J48graft Combined with Sampling Selection Techniques for the Diagnosis of Type 2 Diabetes Mellitus in the PIMA Indian Dataset, Informatics in Medicine Unlocked, 2, pp. 92-104, 2016.

[7] Nawaz Khan, Dhara Gaurav and Thomas Kandl; Performance Evaluation of Levenberg-Marquardt Technique in Error Reduction for Diabetes Condition Classification, Procedia Computer Science, 18, pp. 2629-2637, 2013.

[8] Stavros Lekkas and Ludmil Mikhailov; Evolving Fuzzy Medical Diagnosis of Pima Indians Diabetes and of Dermatological Diseases, Artificial Intelligence in Medicine, 50, pp. 117-126, 2010.

[9] MIMIC; MIMIC-III Database, URL: https://mimic.physionet.org/about/mimic/, Last Accessed by Feb 26 ${ }^{\text {th }}, 2018$.

[10] Nongyao Nai-arun and Rungruttikarn Moungmai; Comparison of Classifiers for the Risk of Diabetes Prediction, Procedia Computer Science, 69, pp. 132-142, 2015.
[11] Mehrbakhsh Nilashi, Othman Ibrahim, Mohammad Dalvi, Hossein Ahmadi and Leila Shahmoradi; Accuracy Improvement for Diabetes Disease Classification: a Case on a Public Medical Dataset, Fuzzy Inf. Eng., 9, pp. 345-357, 2017.

[12] Ahmed Hamza Osman and Hani Moetque Aljahdali; Diabetes Disease Diagnosis Method Based on Feature Extraction Using K-SVM, International Journal of Advanced Computer Science and Applications, 8(1), pp. 236-244, 2017.

[13] Margit Pohl, Sylvia Wiltner, Alexander Rind, Wolfgang Aigner, Silvia Miksch, Thomas Turic and Felix Drexler; Patient Development at a Glance: an Evaluation of a Medical Data Visualization, IFIP Conference on Human-Computer Interaction, LNCS 6949, pp. 292-299, 2011.

[14] Sushant Ramesh, H. Balaji, N. Ch. S. N Iyengar and Ronnie D. Caytiles; Optimal Predictive Analytics of Pima Diabetics Using Deep Learning, International Journal of Database Theory and Application, 10(9), pp. 47-62, 2017.

[15] T. Santhanam and M.S. Padmavathi; Application of K-Means and Genetic Methods for Dimension Reduction by Integrating SVM for Diabetes Diagnosis, Procedia Computer Science, 47, pp. 76-83, 2015.

[16] Nesma Settouti, M. Amine Chikh and Meryem Saidi; Generating Fuzzy Rules for Constructing Interpretable Classifier of Diabetes Disease, Australas Phys Eng Sci Med, pp. 1-14, 2012.

[17] Jack W. Smith, JE Everhart, WC Dickson, WC Knowler and RS Johannes; Using the ADAP Learning Method to Forecast the Onset of Diabetes Mellitus, Annu Comput Appl Med Care, pp. 261-265, 1988.

[18] Grigorios Tzortzis and Aristidis Likas; The Global Kernel k-Means Clustering Method, The 2008 International Joint Conference on Neural Networks, pp. 1978-1985, 2008.

[19] Kamadi V.S.R.P. Varma, Allam Appa Rao, Thummala Sita Mahalakshmi and P.V. Nageswara Rao; A Computational Intelligence Technique for the Effective Diagnosis of Diabetic Patients Using Principal Component Analysis (PCA) and Modified Fuzzy SLIQ Decision Tree Approach, Applied Soft Computing, 49, pp. 137-145, 2016.

[20] World Health Organization (WHO); Diabetes, URL: http://www.who.int/mediacentre/factsheets/fs312/e n/, Last Accessed by Feb 19 ${ }^{\text {th }}, 2018$. 